Pacific Journal of Mathematics

SPECTRAL APPROXIMATION THEOREMS IN LOCALLY

V ON 


\title{
SPECTRAL APPROXIMATION THEOREMS IN LOCALLY CONVEX SPACES
}

\author{
VOLKER WROBEL
}

We present some results on collectively compact operator approximation theory in locally convex Hausdorff spaces (l.c.s.). The notion of a collectively compact family of operators acting on a Banach space has been introduced by Anselone and Palmer in connection with the numerical solution of integral equations. Meanwhile collectively compact families of operators have been studied in general topological vector spaces. In contrast to those investigations dedicated to the characterization of collectively compact families of operators the present paper focuses on spectral approximation theorems in l.c.s. similar to those given by Anselone and Palmer in the case of Banach spaces. In doing this it turns out that the notion of the spectrum, which causes no problems in Banach algebra theory, entails some difficulty. A way out is indicated by using notions and tools of locally convex algebra theory.

o. Notations. Throughout this paper let $E$ denote a l.c.s. over the field of complex numbers $\mathbf{C}$. $E$ is always assumed to be equipped with a basis $\mathscr{P}$ of continuous seminorms $p$. By $\mathscr{U}_{p}$ we denote the closed, convex, and circled neighborhood of zero $\{x \in E: p(x) \leqq 1\}$ in $E$. Let $\mathscr{L}_{s}(E), \mathscr{L}_{c}(E)$ and $\mathscr{L}_{b}(E)$ denote the locally convex algebra of all continuous linear operators on $E$ equipped with the topology of uniform convergence on finite, compact, and bounded subsets of $E$.

The formulation of spectral approximation theorems requires some remarks on the notion of spectrum. In contrast to Banach algebra theory there are different ways for introducing a spectrum for the elements of a locally convex algebra, which in general lead to different sets. For $T \in \mathscr{L}_{b}(E)$ a straightforward generalization from the theory of Banach algebras would lead to the following notions: Denote by $\rho_{B}(T):=\left\{z \in \mathbf{C}:\left(z \cdot \mathrm{id}_{E}-T\right)^{-1} \in \mathscr{L}(E)\right\} \quad$ resp. $\quad \sigma_{B}(T):=\mathbf{C} \backslash \rho_{B}(T)$ the Banach-resolvent set resp. the Banach-spectrum of $T$. These notions which are of great importance for solving eigenvalue problems for the linear operator $T$ unfortunately are not suitable for involving such a powerful tool as the analytic functional calculus for general l.c.s. $E$. That is why we introduce the following notions current in locally convex algebra theory (see [1]). For $T \in \mathscr{L}_{b}(E)$, the spectrum $\sigma(T)$ of $T$ is the complement in the Riemann sphere $\hat{\mathbf{C}}$ of the largest open set $\rho(T)$ in which $z \mapsto R(z, T):=\left(z \cdot \mathrm{id}_{E}-T\right)^{-1}$ is locally holomorphic (in the 
sense of convergence in $\left.\mathscr{L}_{b}(E)\right) . R(\cdot, T)$ is called resolvent, $\rho(T)$ the resolvent set of $T$. For $z_{1}, z_{2} \in \rho_{B}(T)$ the resolvent equation is fulfilled:

$$
R\left(z_{1}, T\right)-R\left(z_{2}, T\right)=\left(z_{2}-z_{1}\right) R\left(z_{1}, T\right) R\left(z_{2}, T\right) .
$$

With these notions of spectrum and resolvent set an analytic functional calculus can be established in the locally convex algebra $\mathscr{L}_{b}(E)$. If $E$ is a Mackey complete l.c.s. and $T$ a precompact or bounded endomorphism on $E$ it can be shown $\sigma(T)=\sigma_{B}(T)$ and $\rho(T)=\rho_{B}(T) \cup\{\infty\}$. In general these sets are different, indeed $\sigma_{B}(T)$ may be empty whereas $\sigma(T)$ never is by Liouville's theorem. For further notations concerning the theory of locally convex spaces the reader is referred to Schaefer's monograph [7].

1. Introduction. In various important applications only approximate solutions of eigenvalue problems for linear operators are computable, the accuracy of which has to be estimated. That is why perturbation theory and spectral approximation theorems entering the scene quite naturally are of particular importance in this field. To begin with we shall give an easy spectral approximation theorem. Although it is less useful in applications, it illustrates the difference between Banach algebra theory and the theory of locally convex algebras so much the better. The proof of the following theorem is easily done by means of Neumann's series and is therefore omitted.

Theorem 1.1. (Uniform Spectral Approximation Theorem) Let E denote a Banach space and $T, T_{n} \in \mathscr{L}(E), n=1,2, \cdots$. Assume $T_{n} \rightarrow T$ in $\mathscr{L}_{b}(E)$. Then for all open subsets $\Omega \subset \hat{\mathbf{C}}$ containing $\sigma(T)$ there exists $n_{0}$ such that

(i) $\quad \sigma\left(T_{n}\right) \subset \Omega$ resp. $\rho\left(T_{n}\right) \supset \hat{\mathbf{C}} \backslash \Omega$ for $n \geqq n_{0}$.

(ii) $R\left(z, T_{n}\right) \rightarrow R(z, T)$ in $\mathscr{L}_{b}(E)$ uniformly for $z \in \hat{\mathbf{C}} \backslash \Omega$.

(iii) The resolvents $R\left(\cdot, T_{n}\right), n \geqq n_{0}$, are equicontinuous on $\hat{\mathbf{C}} \backslash \Omega$.

These statements no longer remain true for general l.c.s. E. This is demonstrated by the following easy

Counterexample. Let $E$ denote the $(F N)$-space $\Pi_{1}^{\infty} \mathbf{C}$ equipped with the usual product topology. Denote by $e_{n}$ the $n$th unit vector of $E$. By $T_{n}, n=1,2, \cdots$ denote the operator $\left(x_{m}\right)_{m \in \mathrm{N}} \mapsto x_{n} e_{n}$. Now one immediately demonstrates $T_{n} \rightarrow 0$ uniformly on $E$, hence we have $T_{n} \rightarrow 0$ in $\mathscr{L}_{b}(E)$ especially. On the other hand $1 \in \sigma_{B}\left(T_{n}\right)=\sigma\left(T_{n}\right)$ for all $n \in \mathbf{N}$.

Putting this into a more general context we cite a result due to Vladimirskii [9] showing that the locally convex algebra $\mathscr{L}_{b}(E)$ is rather 
pathological and that the counterexample does represent the rule for nonnormable 1.c.s. $E$.

THEOREM 1.2. Let E denote any given l.c.s. Then the following two statements are equivalent

(i) $E$ is a nonnormable l.c.s.

(ii) For every neighborhood $\mathscr{W}$ of zero in $\mathscr{L}_{b}(E)$ there exists $T \in \mathscr{W}$ such that $\mathrm{id}_{E}+T$ is not injective.

A reformulation of (ii) yields

(ii)' $^{\prime}$ For every neighborhood $\mathcal{W}$ of zero in $\mathscr{L}_{b}(E)$ there exists $T \in \mathscr{W}$ such that $(-1)$ is an eigenvalue of $T$.

As already mentioned Theorem 1.1 is less useful in practice. First, checking whether a sequence of operators $T_{n}$ converges in $\mathscr{L}_{b}(E)$ is very difficult in general if possible at all. Second, in many applications this is not even true. In practice one checks whether a sequence $T_{n}$ converges pointwise. Then of course additional assumptions have to be made to ensure results similar to the statements of Theorem 1.1. In important applications (see [3]) $\left\{T-T_{n}: n \in \mathbf{N}\right\}$ is collectively compact in the sense of the following

Definition. A family $\mathscr{H} \subset \mathscr{L}(E)$ is collectively compact if there exists a neighborhood $\mathcal{U}_{p}$ of zero in $E$ such that $\cup_{T \in \mathscr{H}} T\left(\mathcal{U}_{p}\right)$ is a relatively compact subset of $E$. For further characterizations and properties of collectively compact sets of linear operators in topological vector spaces the reader is referred to [4], [5]. Finally we mention that the statements 3.1, 3.2, and 3.3 of [4] are in general not true as our counterexample following Theorem 1.1 demonstrates.

2. Spectral approximation theorems. For the proof of our main theorem the following easy lemma is needed.

LEMma. Let $E, F$, and $G$ denote three l.c.s., $B$ any bounded subset of $E, \mathscr{U}_{p}$ and $\mathscr{V}_{q}$ neighborhoods of zero in $F$ and $G$. For $T \in \mathscr{L}(E, F)$ and $S \in \mathscr{L}(F, G)$ we have the following inequality

$$
\sup _{x \in B} q(S \circ T x) \leqq \sup _{x \in B} p(T x) \sup _{y \in \mathscr{U}_{p}} q(S y)
$$

Proof. For all $\lambda>0$ such that $T B \subset \lambda U_{p}$ the following inequality is true

$$
\sup _{x \in B} q(S \circ T x) \leqq \sup _{y \in \lambda \mathscr{U}_{p}} q(S y)=|\lambda| \sup _{y \in \mathscr{U}_{p}} q(S y) .
$$


Hence for $\lambda_{0}:=\inf \left\{\lambda>0: T B \subset \lambda U_{p}\right\}=: \sup _{x \in B} p(T x)$. The right hand side may be $+\infty$.

For the rest of this section let $E$ be a barreled, sequentially complete l.c.s. These assumptions on $E$ form a natural limit to which one can hope to generalize the theorems of Anselone and Palmer without rather artificial conditions. First, the Banach-Steinhaus theorem plays a vital role in the demonstration of the spectral approximation theorems. Second, in dealing with resolvents mostly Cauchy series are involved and the given assumption on $E$ ensures that $\mathscr{L}_{b}(E)$ is sequentially complete. Third, holomorphic functions with values in $\mathscr{L}_{b}(E)$ can be handled easily:

A function $H$ from a domain $G$ of the Riemann sphere with values in $\mathscr{L}_{b}(E)$ is holomorphic iff for all $x \in E$ and $\varphi \in E^{\prime}$ the complex valued function $z \mapsto\langle\varphi, H(z) x\rangle$ is holomorphic in $G$. This, too, is a consequence of the Banach-Steinhaus theorem.

The following theorem is a locally convex version of a spectral approximation theorem due to Anselone and Palmer [2] in the case of Banach spaces.

Theorem 2.1. Let $T, T_{n} \in \mathscr{L}(E), n=1,2, \cdots$. Assume $T_{n} \rightarrow T$ in $\mathscr{L}_{s}(E)$ and $\left\{T_{n}-T: n \in \mathbf{N}\right\}$ collectively compact. Then the following is true:

For every open subset $\Omega \subset \hat{\mathbf{C}}$ containing $\sigma(T)$ there exists $n_{0} \in \mathbf{N}$ such that

(i) $\quad \sigma\left(T_{n}\right) \subset \Omega$ resp. $\rho\left(T_{n}\right) \supset \hat{\mathbf{C}} \backslash \Omega$ for $n \geqq n_{0}$.

(ii) $R\left(z, T_{n}\right) \rightarrow R(z, T)$ in $\mathscr{L}_{s}(E)$ uniformly for $z \in \hat{\mathbf{C}} \mid \Omega$.

(iii) $\left\{R\left(z, T_{n}\right)-R(z, T): n \geqq n_{0}, z \in \hat{\mathbf{C}} \backslash \Omega\right\}$ is collectively compact.

(iv) The resolvents $R\left(\cdot, T_{n}\right)$ are equicontinuous on $\hat{\mathbf{C}} \backslash \Omega$ for $n \geqq n_{0}$.

Proof. For an abbreviation let $S_{n}(z, T):=R(z, T)\left(T_{n}-T\right)$. Since $R(\cdot, T)$ is locally holomorphic on $\hat{\mathbf{C}} \mid \Omega$ the set $\{R(z, T): z \in \hat{\mathbf{C}} \mid \Omega\}$ is compact in $\mathscr{L}_{b}(E)$. By [4], Prop. 2.3, $\mathscr{H}:=\left\{S_{n}(z, T): n \in \mathbf{N}, z \in \hat{\mathbf{C}} \backslash \Omega\right\}$ is collectively compact. Hence we find a neighborhood $\mathcal{U}_{p_{0}}$ of zero such that $\mathscr{H} u_{p_{0}}$ is relatively compact. Now by the Banach-Steinhaus theorem we can find $n_{0}$ such that

$$
\left(S_{n}(z, T)\right)^{2} \mathcal{U}_{p_{0}} \subseteq R(z, T)\left(T_{n}-T\right) \mathscr{H} \mathcal{U}_{p_{0}} \subseteq \frac{1}{2} \mathcal{U}_{p_{0}} \text { for } n \geqq n_{0}
$$

uniformly for $z \in \hat{\mathbf{C}} \backslash \Omega$.

Now for any given bounded set $B \subset E$ and neighborhood of zero $U_{p_{1}}$ of $E, i \in \mathbf{N}$ and $i \geqq 2$ we get the following inequality by means of the above lemma

$$
\text { (**) } \sup _{x \in B} p_{1}\left(S_{n}(z, T)^{i} x\right) \leqq \sup _{x \in B} p_{0}\left(S_{n}(z, T)^{1-2} z\right) \sup _{y \in \mathcal{U}_{p_{0}}} p_{1}\left(S_{n}(z, T)^{2} y\right) .
$$


As $\left\{S_{n}(z, T)^{2}: n \in \mathbf{N}, z \in \hat{\mathbf{C}} \backslash \Omega\right\} \mathcal{U}_{p_{n}}$ is a relatively compact subset of $E$ (**) together with $(*)$ implies that $\sum_{i=2}^{\infty} S_{n}(z, T)^{i}$ is Cauchy and hence convergent in $\mathscr{L}_{b}(E)$ uniformly for $z \in \hat{\mathbf{C}} \mid \Omega$ and $n \geqq n_{0}$. Hence the limit function $\quad z \mapsto\left(\mathrm{id}_{E}-S_{n}(z, T)\right)^{-1}=R\left(z, T_{n}\right)\left(z \cdot \mathrm{id}_{E}-T\right) \quad$ is locally holomorphic on $\hat{\mathbf{C}} \mid \Omega$ as a function with values in $\mathscr{L}_{b}(E)$. But then $R\left(\cdot, T_{n}\right)$ is locally holomorphic too, considered as a function with values in $\mathscr{L}_{b}(E)$. Thus (i) has been shown. Furthermore $\sum_{i=2}^{\infty} S_{n}(z, T)^{i} \rightarrow 0$ in $\mathscr{L}_{b}(E)$ uniformly for $z \in \hat{\mathbf{C}} \backslash \Omega$ by $(*)$ and $(* *)$. So we get

$$
R\left(z, T_{n}\right)=\left(\operatorname{id}_{E}+S_{n}(z, T)+\sum_{i=2}^{\infty} S_{n}(z, T)^{i}\right) R(z, T) \rightarrow R(z, T)
$$

in $\mathscr{L}_{s}(E)$ uniformly for $z \in \hat{\mathbf{C}} \backslash \Omega$, and hence (ii).

Because of (ii) given $x \in E$ and a neighborhood of zero $\mathcal{U}_{p}$ in $E$ there exists $n_{1}$ such that

$$
\left\{R\left(z, T_{n}\right) x: z \in \hat{\mathbf{C}} \mid \Omega, n \geqq n_{1}\right\} \subset\{R(z, T) x: z \in \hat{\mathbf{C}} \mid \Omega\}+U_{p} .
$$

Hence $\left\{R\left(z, T_{n}\right): z \in \hat{\mathbf{C}} \backslash \Omega, n \geqq n_{1}\right\}$ is equicontinuous by the BanachSteinhaus theorem. Now an easy calculation gives $R(z, T)-R\left(z, T_{n}\right)=$ $R(z, T)\left(T-T_{n}\right) R\left(z, T_{n}\right)$. Using this we get (iii), because $\{R(z, T)$ $\left.\left(T-T_{n}\right): z \in \hat{\mathbf{C}} \mid \Omega, n \in \mathbf{N}\right\}$ is collectively compact.

As equicontinuous sets of $\mathscr{L}_{b}(E)$ are bounded in $\mathscr{L}_{b}(E)$ (see [7], p. 83 ) (iv) will yield from the following inequality by means of the resolvent equation for $R\left(\cdot, T_{n}\right)$ and the lemma: Let $B$ be any given bounded subset of $E$ and $p_{1}$ a continuous seminorm of $E$ and $p_{2}$ such that $\left\{R\left(z, T_{n}\right): z \in \hat{\mathbf{C}} \backslash \Omega, n \geqq n_{1}\right\} \mathcal{U}_{p_{2}} \subseteq \mathcal{U}_{p_{1}}$. Then

$$
\begin{aligned}
& \sup _{x \in B} p_{1}\left(\left(R\left(z_{1}, T_{n}\right)-R\left(z_{2}, T_{n}\right)\right) x\right)=\left|z_{1}-z_{2}\right| \sup _{x \in B} p_{1}\left(R\left(z_{1}, T_{n}\right) R\left(z_{2}, T_{n}\right) x\right) \\
& \leqq\left|z_{1}-z_{2}\right| \sup _{x \in B} p_{2}\left(R\left(z_{2}, T_{n}\right) x\right) \sup _{y \in \mathscr{M _ { P }}} p_{1}\left(R\left(z_{1}, T_{n}\right) y\right) .
\end{aligned}
$$

As $\left\{R\left(z, T_{n}\right): z \in \hat{\mathbf{C}} \backslash \Omega, n \geqq n_{1}\right\}$ is bounded in $\mathscr{L}_{b}(E)$ we are done.

The rest of this section is devoted to an adaptation of Theorem 2.1 to practical applications. Of course the most important question has to be answered how eigenvalues and eigenmanifolds of $T$ can be obtained from their approximations defined in terms of the approximating sequence $\left(T_{n}\right)_{n \in \mathrm{N}}$. The main tool for doing this is the analytic functional calculus. 
But first we shall give a result following at once from Theorem 2.1.

COROllary 2.2. Let the hypotheses of 2.1 be fulfilled. Then the following is true

(i) If $z_{0} \in \hat{\mathbf{C}}$ is a cluster point of $\left\{z_{n}: n \in \mathbf{N}, z_{n} \in \sigma\left(T_{n}\right)\right\}$ then $z_{0} \in \sigma(T)$.

(ii) If $z_{0}$ is an isolated point of $\sigma(T)$, then there exists a sequence $\left(z_{n}\right)_{n \in \mathrm{N}}, z_{n} \in \sigma\left(T_{n}\right)$, such that $z_{n} \rightarrow z_{0}$.

Now we shall sketch some notions from the analytic functional calculus in locally convex algebras (see Allan [1] for details). Roughly speaking it turns out that the functional calculus for closed endomorphisms on Banach spaces due to Taylor (see [8]) admits a generalization for continuous linear operators on a locally convex space.

Definition 2.3. By a Cauchy domain we denote an open subset $\Delta$ of $\hat{\mathbf{C}}$ such that $\Delta$ consist of a finite number of components the closures of which are pairwise disjoint, the boundary $\partial \Delta$ of $\Delta$ consists of a finite number of closed rectifiable Jordan curves contained in $\mathbf{C}$, no two of which intersect.

For $T \in \mathscr{L}_{b}(E)$ let $\mathscr{H}_{x}(T)$ denote the set of all germs of functions being locally holomorphic on $\sigma(T) \cup\{\infty\}$. For every $f \in \mathscr{H}_{\infty}(T)$ there exists a Cauchy domain $\Delta_{f}$ such that $\sigma(T) \subset \Delta_{f} \subset \bar{\Delta}_{f} \subset D(f)$ (we do not distinguish between the germ $f$ and a function $f$ representing the germ, thus $D(f)$ denotes the domain of a representing function being locally holomorphic on $D(f))$. The mapping

$$
\mathscr{H}_{x}(T) \ni f \mapsto f(T):=f(\infty) \mathrm{id}_{E}+(2 \pi i)^{-1} \int_{\partial \Delta_{f}} f(z) R(z, T) d z
$$

defines an algebra homomorphism from $\mathscr{H}_{x}(T)$ into $\mathscr{L}_{b}(E)$ (being continuous if $\mathscr{H}_{x}(T)$ is equipped with the usual inductive limit topology). With these notions the following theorem can be derived from Theorem 2.1 by similar arguments used by Anselone [3] for the derivation of his Theorem 4.15. The proof is therefore omitted.

THEOREM 2.4. Let the hypotheses of 2.1 be fulfilled. Then for every $f \in \mathscr{H}_{x}(T)$ we find $n_{0} \in \mathbf{N}$ such that for $n \geqq n_{0}$ we have

(i) $f \in \mathscr{H}_{x}\left(T_{n}\right)$.

(ii) $f\left(T_{n}\right) \rightarrow f(T)$ in $\mathscr{L}_{s}(E)$.

(iii) $\left\{f(T)-f\left(T_{n}\right): n \geqq n_{0}\right\}$ is collectively compact.

Let $\sigma \subset \sigma(T)$ denote a spectral set, that means $\sigma$ is both open and closed relatively $\sigma(T)$. Let $\pi_{\sigma}(\cdot) \in \mathscr{H}_{\infty}(T)$ such that $\pi_{\sigma}(z)=1$ for $z \in \sigma$ 
and $\pi_{\sigma}(z)=0$ for $z \in \sigma(T) \backslash \sigma$. Then $\pi_{\sigma}(T)$ is a projection by the functional calculus, called the spectral projection belonging to $\sigma$. For the further discussion let us assume the hypotheses of 2.1 are fulfilled. Denote by $U$ an open neighborhood of $\sigma$ being disjoint from $\sigma(T) \backslash \sigma$. Then $\sigma_{n}:=\left\{z \in \mathcal{U}: z \in \sigma\left(T_{n}\right)\right\}$ is a spectral set of $\sigma\left(T_{n}\right)$ for $n$ sufficiently large by Theorem 2.1 (i). Let $\pi_{\sigma}\left(T_{n}\right):=\pi_{\sigma_{n}}\left(T_{n}\right)$. By Theorem 2.4. (iii) $\left\{\pi_{\sigma}(T)-\pi_{\sigma}\left(T_{n}\right): n \geqq n\right\}$ is collectively compact. If one of the spectral projections is compact then all are compact mappings by [4] Prop. 2.2. Hence $\operatorname{dim} \pi_{\sigma}\left(T_{n}\right) E<\infty$ for $n \geqq n_{0}$. By transition to a suitable Banach space the spectral projections factoring through this space, we are allowed to apply Theorem 4.13 of Anselone [3] to $\left\{\pi_{\sigma}\left(T_{n}\right): n \geqq n_{0}\right\} \cup\left\{\pi_{\sigma}(T)\right\}$. Identifying all infinite cardinals additionally we get the following

THEOREM 2.5. Let $\pi_{\sigma}\left(T_{n}\right) \rightarrow \pi_{\sigma}(T)$ in $\mathscr{L}_{s}(E)$ and $\left\{\pi_{\sigma}\left(T_{n}\right)-\pi_{\sigma}(T)\right.$ : $\left.n \geqq n_{0}\right\}$ collectively compact. Then $\operatorname{dim} \pi_{\sigma}\left(T_{n}\right) E=\operatorname{dim} \pi_{\sigma}(T) E$ for $n$ sufficiently large.

If $z_{0}$ is a pole of the resolvent $z \mapsto R(z, T)$ and the spectral projection $\pi_{\{z 0\}}(T)$ is of finite rank then $z_{0}$ is called a pole of finite multiplicity. It can be shown that if $z_{0}$ is an isolated point of $\sigma(T)$ and $\pi_{\{z 0\}}(T)$ of finite rank then $z_{0}$ is a pole of finite multiplicity.

For example all eigenvalues of a compact operator being different from 0 are poles of finite multiplicity. After this remarks the following theorem is quite clear.

THEOREM 2.6. Assume the hypotheses of 2.1 are fulfilled. Then

(i) If $z_{0}$ is a pole of finite multiplicity for $z \mapsto R(z, T)$ then there exists a sequence $\left(z_{n}\right)_{n \in \mathrm{N}}, z_{n}$ being a pole of finite multiplicity for $z \mapsto R\left(z, T_{n}\right)$ such that $z_{n} \rightarrow z_{0}$.

(ii) Assume $z_{0}$ is an isolated point of $\sigma(T)$. Let $z_{n} \rightarrow z_{0}, z_{n} \in \sigma\left(T_{n}\right)$ being a pole of finite multiplicity for $z \mapsto R\left(z, T_{n}\right)$ (n sufficiently large of course!). Then $z_{0}$ is a pole of finite multiplicity for $z \mapsto R(z, T)$.

Finally we shall discuss the approximation of eigenmanifolds. We begin with a rather simple case, also discussed by Anselone [3].

THEOREM 2.7. Assume the hypotheses of 2.1 are fulfilled. Let $z_{0} \in \sigma(T)$ be an isolated point and $\operatorname{dim} \pi_{\{z 0\}}(T) E=1$. Then there exists $x \in E$ such that $T x=z_{0} x, \pi_{\left\{z_{0}\right\}}(T) E=\operatorname{span}\{x\}, \pi_{\left\{z_{0}\right\}}(T) x=x$.

For each $n$ sufficiently large there exists $z_{n}$ such that $\sigma_{n}=\left\{z_{n}\right\}, z_{n} \rightarrow z_{0}$.

Let $x_{n}:=\pi_{\{z 0\}}\left(T_{n}\right) x$. Then $x_{n} \rightarrow x$ and $T_{n} x_{n}=z_{n} x_{n}$.

For $n$ sufficiently large we have $x_{n} \neq 0$ and $\pi_{\{z\}}\left(T_{n}\right) E=\operatorname{span}\left\{x_{n}\right\}$. 
Proof. All we have to do is showing $T_{n} x_{n}=z_{n} x_{n}$. As $x_{n}=$ $\pi_{\{z 0\}}\left(T_{n}\right) x \rightarrow \pi_{\{z\}\}}(T) x=x$ by Theorem 2.4 (iii) we have $x_{n} \neq 0$ for $n$ sufficiently large. Hence by Theorem $2.5 \pi_{\{z 0\}}\left(T_{n}\right) E=\operatorname{span}\left\{x_{n}\right\}$ and therefore $T_{n} x_{n}=z_{n} x_{n}$.

A more general situation is considered in the following.

THEOREM 2.8. Assume the hypotheses of 2.1 are fulfilled. Let $\sigma$ denote a spectral set of $\sigma(T)$ and $\operatorname{dim} \pi_{\sigma}(T) E<\infty$. By $\mathcal{U}_{p}$ denote a neighborhood of zero in $E$ such that $\left\{\pi_{\sigma}\left(T_{n}\right): n \geqq n_{0}\right\} U_{p}=: K$ is a relatively compact subset of $E$.

For $x_{n} \in \pi_{\sigma}\left(T_{n}\right) E$ and $p\left(x_{n}\right) \leqq 1, n$ sufficiently large we have $x_{n}-\pi_{\sigma}(T) x_{n} \rightarrow 0$ in $E$ for $n \rightarrow \infty$.

Proof. Since $\pi_{\sigma}(T)-\pi_{\sigma}\left(T_{n}\right)$ converging pointwise by Theorem 2.4 (ii) to zero also converges to zero in $\mathscr{L}_{c}(E)$ by the Banach-Steinhaus theorem, the theorem is an immediate consequence of the following identity, since $K$ is relatively compact:

$$
\begin{aligned}
x_{n}-\pi_{\sigma}(T) x_{n} & =\pi_{\sigma}\left(T_{n}\right) x_{n}-\pi_{\sigma}(T) \circ \pi_{\sigma}\left(T_{n}\right) x_{n} \\
& =\left(\pi_{\sigma}\left(T_{n}\right)-\pi_{\sigma}(T)\right) \circ \pi_{\sigma}\left(T_{n}\right) x_{n} .
\end{aligned}
$$

REMARK. If $T$ is a bounded operator on a Mackey complete l.c.s. and $\left\{T-T_{n}: n \in \mathbf{N}\right\}$ collectively compact then all $T_{n}$ are bounded operators too. Then by transition to a suitable Banach space one gets the whole theory of Anselone and Palmer without tools of locally convex algebra theory. How the spectral theory of bounded operators on a Mackey complete 1.c.s. is reduced to the classical case of continuous operators on Banach spaces can be deduced for example from Pietsch [6].

\section{REFERENCES}

1. G. R. Allan, A spectral theory for locally convex algebras, Proc. London Math. Soc., (3) 15 (1965), 399-421.

2. P. M. Anselone, T. W. Palmer, Spectral analysis of collectively compact, strongly convergent operator sequences, Pacific J. Math., 25 (1968), 423-431.

3. P. M. Anselone, Collectively Compact Operator Approximation Theory, Prentice Hall 1971.

4. J. D. DePree, J. A. Higgins, Collectively compact sets of linear operators, Math. Z., 115 (1970), $366-370$.

5. J. D. DePree, H. S. Klein, Characterization of collectively compact sets of linear operators, Pacific J. Math., 55 (1974), 45-54.

6. A. Pietsch, Zur Fredholmschen Theorie in lokalkonvexen Räumen, Studia Math., 22 (1963), 161-179. 
7. H. H. Schaefer, Topological vector spaces, Springer Graduate texts in Mathematics $\mathbf{3}$ New York 1970.

8. A. E. Taylor, Introduction to Functional Analysis, Wiley \& Sons New York 1958.

9. Ju. N. Vladimirskii, Kriterien der Normierbarkeit eines lokalkonvexen Raumes, Mat. Issled, 20 (1971), 138-141 (Russian), Zentralblatt 1974 Bd. 257 No. 46007.

Received January 28, 1975 and in revised form January 12, 1976.

MathematisChes Seminar DER

Christian-Albrechts-UNIVERSITÄT

D-23 KIEL 1

FED. REP. GERMANY 




\section{Pacific Journal of Mathematics}

Vol. 64, No. 1

May, 1976

Walter Allegretto, Nonoscillation theory of elliptic equations of order $2 n \ldots \ldots \quad 1$

Bruce Allem Anderson, Sequencings and starters.................. 17

Friedrich-Wilhelm Bauer, A shape theory with singular homology .......... 25

John Kelly Beem, Characterizing Finsler spaces which are

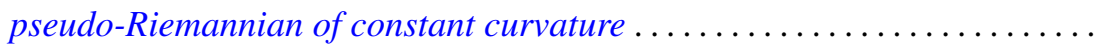

Dennis K. Burke and Ernest A. Michael, On certain point-countable

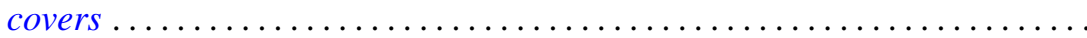

Robert Chen, A generalization of a theorem of Chacon ............... 93

Francis H. Clarke, On the inverse function theorem ................ 97

James Bryan Collier, The dual of a space with the Radon-Nikodým

property ....................................... 103

John E. Cruthirds, Infinite Galois theory for commutative rings ............ 107

Artatrana Dash, Joint essential spectra......................... 119

Robert M. DeVos, Subsequences and rearrangements of sequences in FK

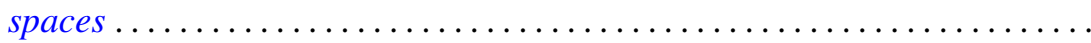

Geoffrey Fox and Pedro Morales, Non-Hausdorff multifunction generalization

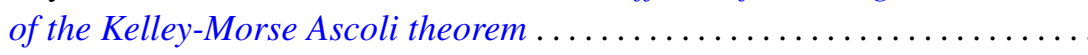

Richard Joseph Fleming, Jerome A. Goldstein and James E. Jamison, One

parameter groups of isometries on certain Banach spaces.............

Robert David Gulliver, II, Finiteness of the ramified set for branched

immersions of surfaces

Kenneth Hardy and István Juhász, Normality and the weak cb property ...... 167

C. A. Hayes, Derivation of the integrals of $L^{(q)}$-functions.

Frederic Timothy Howard, Roots of the Euler polynomials .

Robert Edward Jamison, II, Richard O'Brien and Peter Drummond Taylor, On

embedding a compact convex set into a locally convex topological vector space ....................................

Andrew Lelek, An example of a simple triod with surjective span smaller than span ...

Janet E. Mills, Certain congruences on orthodox semigroups

Donald J. Newman and A. R. Reddy, Rational approximation of $e^{-x}$ on the positive real axis.

John Robert Quine, Jr., Homotopies and intersection sequences ...

Nambury Sitarama Raju, Periodic Jacobi-Perron algorithms and fundamental units ....

Herbert Silverman, Convexity theorems for subclasses of univalent functions. . .

Charles Frederick Wells, Centralizers of transitive semigroup actions and endomorphisms of trees.........................

Volker Wrobel, Spectral approximation theorems in locally convex spaces ..................... 